\title{
Signals of Autodyne Sensors with Sinusoidal Frequency Modulation
}

\author{
Vladislav Ya. NOSKOV ${ }^{1}$, Kirill A. IGNATKOV ${ }^{1}$, Andrey P. CHUPAHIN ${ }^{1}$, \\ Aleksandr S. VASILYEV ${ }^{2}$, Gennadiy P. ERMAK ${ }^{2}$, Sergey M. SMOLSKIY ${ }^{3}$ \\ ${ }^{1}$ Dept. of Radioelectronics and Communications, Ural Federal Univ. (UrFU), Mira St., 19, 620002, Ekaterinburg, Russia \\ ${ }^{2}$ O.Ya. Usikov Inst. for Radio Physics and Electronics (IRE NASU), Acad. Proscury St., 12, 61085, Kharkiv, Ukraine \\ ${ }^{3}$ Dept. of Radio Signals Generation and Processing, National Research University "Moscow Power Engineering Institute" \\ (NRU “MPEI”), Krasnokazarmennaya St., 14, 111250, Moscow, Russia
}

noskov@oko-ek.ru, ermak@ire.kharkov.ua,smolskiysm@mail.ru

Submitted March 17, 2017 / Accepted October 26, 2017

\begin{abstract}
Research results of signal formation features of the autodyne sensor (AS) with sinusoidal frequency modulation (FM) are presented. Expressions for low frequency information signals are given and their numerical analysis is performed for cases when signal period is substantially longer than reflected radiation delay time as well as for the case when this inequality is not fulfilled. It is proved that at a small distance to the reflected object when feedback parameter value is commensurable with 1, the distortions of FM AS signals are typically caused by reflected radiation phase incursion irregularities. When distance grows the distortion level becomes significantly smaller due to delay phenomenon of reflected radiation. Experimental research results are obtained on the example of the autodyne oscillator based on the 8-mm wavelength range Gunn diode with varicap-based FM.
\end{abstract}

\section{Keywords}

Autodyne, autodyne signals, autodyne sensor, frequency modulation, Gunn-diode oscillator

\section{Introduction}

Among modern radar devices of very different practical application, we can distinguish the short-range radars (SRRs) as a separate radar type which functions in specific conditions. Besides standard task of target detection on short distances, they perform the function of target movement parameter measurement [1-4]. The following requirements are imposed on SRR: the extremely high processing speed of the radar signal, high detection reliability and operation accuracy, small dimensions and low cost at mass-production. The so-called last-mile radars can also be attributed to the requirement of increased robustness to the impact of active and passive interferences.

Apart from military application, such radars find the wide utilization as reliable and imperceptible sensors in guarding systems [5], anti-collision sensors for transport, for instance, for cars, including radars for observation of latency car zones and their parking in dense rural conditions [2], [3]. In railway transport, they can be used as measuring systems for car motion parameters in hump yards of large railway junctions, in occupation detectors of pointworks and railway crossings [6]. Radar sensors are used in equipment for biomedical and scientific research as sensors in robotics and for technological process measuring in industry as well as real time and non-contact diagnostics of turbo-aggregates etc. (see, for instance, [2-4], [7-10]).

To reduce dimensions and cost of the radar system and especially its front-end, it is preferable to use highfrequency unit of millimeter-wavelength range and to combine transmitter-receiver functions in a single unit a transceiver. The transceiver can be designed with completely separate chains of the transmitter and the receiver with separate antennas. In this case to design the transceiver, the super-heterodyne or direct-conversion types of architecture are widely used to shift the reflected signal into the range of low or 'zero' frequencies. The latter architecture is called homodyne [11].

At the same time, there is another well-known transceiver architecture called 'autodyne' of 'self-oscillating mixing' [12-14]. In this architecture, the radio front-end is designed on the basis of the only stage - an oscillator (an autodyne) which combines the functions of the probing signal transmitter and the reflected signal receiver. Such architecture simplifies the unit design, provides its compactness and relatively low cost of the transceiver module.

At present, autodyne modules including oscillatingemitting ones with different topology are manufactured in the form of monolithic and hybrid integrated circuits [15-17]. Advantages of these devices are high sensitivity of initial data conversion into the output information signal as well as contact-free and practical inertia-absent measurements of various parameters and characteristics of the objects to be located. 
Operation principle of these devices is based on the autodyne effect which consists in the variations of oscillation parameters (RF process amplitude, its power, frequency and phase as well as all DC currents and bias voltages of the active element) under the influence of its own reflected radiation. This effect is manifested in all types of oscillators in the frequency range from $\mathrm{RF}$ to the optical range [1], [18-20]. Registration of these variations in the form of autodyne signals and their further processing ensures the possibility of obtaining information about the reflected object and parameters of its relative motion.

The application of various types of signal modulation (amplitude, frequency, pulse) and received signal processing substantially increase the AS functionality $[1,21$, 22]. Autodyne transceivers with different modulation types have the following advantages: high AS sensitivity in case of detection of location objects on the background of reflections from an underlying terrain, the operation reliability in complicated meteorological conditions, the possibility of object selection at given distances and others.

Among the above mentioned modulation types, the frequency modulation (FM) has become the most popular [1, 23-26]. As in the usual SRR of the homodyne type, the linear and sinusoidal FM in AS found the widest application. Sinusoidal FM is the simplest and therefore widely spread. Its application does not require very strict linearity of the modulation characteristic and it is possible to process signals easily via filtering. Advantages of sinusoidal FM are the relatively low higher harmonic level of the oscillator parasitic amplitude modulation (PAM), simplicity of modulation function formation and extraction of weak signals on the background of the main components of the PAM spectrum. Sinusoidal FM provides a possibility to form zones of target selection in range, to separately measure the reflecting object range and velocity as well as direction of its movement.

A large number of publications are devoted to FM AS signal formation features. These papers deal with different mathematical models of autodynes and various representations of impact functions of the proper reflected radiation on the autodyne oscillator. However, at present the equation solution for the developed FM AS model is obtained only for the first approximation of the retarded impact function, which is true under the following condition: $\tau<<T_{\mathrm{a}}$ where $\tau$ is the delay time of the reflected radiation; $T_{\mathrm{a}}$ is the period of the low frequency autodyne signal [24-27]. This inequation may be violated in practice. For instance, in 8-mm wavelength range when frequency deviation is $500 \mathrm{MHz}$ [23], frequency of sinusoidal FM is $10 \mathrm{kHz}$ and the distance to the location object is $75 \mathrm{~m}, \tau$ is $0.5 \times 10^{-6} \mathrm{sec}$, while $T_{\mathrm{a}}$ is $0.2 \times 10^{-6} \mathrm{sec}$.

The aim of the present paper is to obtain the general solution of retarded equation system for the single-circuit FM oscillator which is under the influence of the proper reflected radiation. Then, on the basis of this solution, it is necessary to consider AS signal formation features at arbitrary delay time of the reflected radiation as well as to analyze the dependence of signal forms and their distortion degree on oscillator modulation parameters and the distance to the location object.

\section{The Main Relations for Signal Analysis}

The equivalent circuit of FM autodyne is presented in Fig. 1. The main components of the circuit are reduced to the one-port active element (AE) section in Fig. 1. In the general case, the averaged (over the RF oscillation period) AE admittance $Y_{\mathrm{AE}}$ is a complex variable depending on the current values of the amplitude $A$ and the frequency $\omega$ : $Y_{\mathrm{AE}} \equiv Y_{\mathrm{AE}}(A, \omega)=G_{\mathrm{AE}}(A, \omega)+\mathrm{j} B_{\mathrm{AE}}(A, \omega)$, where $G_{\mathrm{AE}}, B_{\mathrm{AE}}$ are $\mathrm{AE}$ resistive and reactive conductance, respectively. The oscillator cavity is presented by the equivalent oscillating circuit which contains the inductance $L_{\text {cav }}$, the proper loss conductance $G_{\text {cav }}$ and the capacitor $C_{\text {cav }}$ (see Fig. 1). The capacitance represents the sum of capacitances of the cavity and the varicap $C_{\mathrm{var}}$ which is used for electronic tuning of the oscillator frequency.

The dependent current source $j_{\mathrm{s}} \equiv j_{\mathrm{s}}(t, \tau)$ is connected in parallel to the oscillating circuit. This source describes that AS is under the influence of the proper reflected radiation which is delayed for time $\tau=2 l / c$ with respect to the current time $t$, where $l$ is the distance to the reflected object, $c$ is radiation speed. The conductance $G_{\mathrm{L}}$ is the oscillator load (an antenna, for instance).

Using the Kirchhoff laws, the differential equations for the circuit in Fig. 1 were obtained for instantaneous values of currents and voltages. Then, applying the methods of slowly-changing amplitudes and averaging in Kurokawa's description [28], the abbreviated differential equations for oscillation amplitude and phase were obtained. After that, equations for the steady-state values of amplitude $A_{0}$ and frequency $\omega_{0}$ of the autonomous oscillator were obtained.

Assuming that the reflected radiation has a small level, using the known approach [29], the system of linearized differential equations is obtained for small relative variations of the amplitude $a=\left(A-A_{0}\right) / A_{0}$ and the frequency $\chi=\left(\omega-\omega_{0}\right) / \omega_{0}$ of oscillations:

$$
\begin{gathered}
\left(Q_{\mathrm{L}} / \omega_{\text {cav }}\right)(\mathrm{d} a / \mathrm{d} t)+\alpha a+\varepsilon \chi=\Gamma(t, \tau) \eta \cos \delta(t, \tau), \\
\beta a+Q_{\mathrm{L}} \chi=-\Gamma(t, \tau) \eta \sin \delta(t, \tau)
\end{gathered}
$$

where $Q_{\mathrm{L}}, \omega_{\text {cav }}$ are the loaded Q-factor and the cavity natural frequency; $\alpha, \varepsilon, \beta$ are dimensionless parameters defining the reduced slope of the increment, non-isodromic property and the oscillator non-isochronity, respectively [29]; $\Gamma(t, \tau)=\Gamma_{0}[A(t, \tau) / A(t)], \delta(t, \tau)=\Psi(t)-\Psi(t, \tau)$ are the modulus and the phase of the reflection factor in voltage reduced to the oscillator output; $\Gamma_{0}$ is the damping factor in voltage at emission propagation to the object and back; $A(t, \tau), \Psi(t, \tau)$ are oscillation amplitudes and phases from 


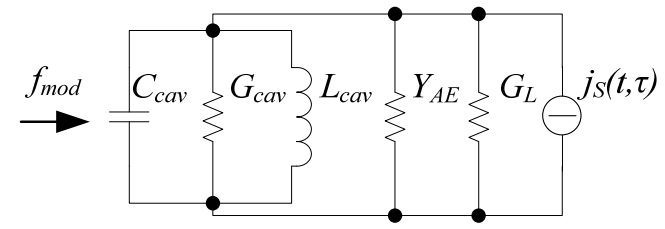

Fig. 1. Equivalent circuit of FM AS.

the system pre-history at $(t-\tau) ; \eta=Q_{\mathrm{L}} / Q_{\mathrm{ex}}, Q_{\mathrm{ex}}$ are efficiency and external Q-factor of the oscillating system; $A(t)$, $\Psi(t)$, are oscillation amplitude and phase in the current moment of time; $G=G_{\text {cav }}+G_{\mathrm{L}}$.

In case FM is achieved by changing bias voltage across varicap, the variations of frequency inevitably happen with variations of oscillation amplitude:

$$
\begin{aligned}
& \omega(t)=\omega_{0}+\Delta \omega_{\mathrm{FM}}(t)=\omega_{0}\left[1+m_{\mathrm{FM}} f_{\mathrm{mod}}(t)\right], \\
& A(t)=A_{0}\left[1+a_{\mathrm{AM}}(t)\right]=A_{0}\left[1+m_{\mathrm{AM}} f_{\mathrm{mod}}(t)\right]
\end{aligned}
$$

where $m_{\mathrm{FM}}=\Delta \omega_{\mathrm{FM}} / \omega_{0}$ and $m_{\mathrm{AM}}=\Delta A_{\mathrm{AM}} / A_{0}$ are coefficients of frequency (FM) and amplitude (AM) modulation, respectively; $\Delta \omega_{\mathrm{FM}}, \Delta A_{\mathrm{AM}}$ are the maximal deviations of oscillation amplitude and frequency from their steady-state values $A_{0}$ and $\omega_{0}$ due to $\mathrm{FM} ; f_{\text {mod }}(t)$ is the normalized modulation function with $\Omega_{\bmod }$ frequency. Quasi-static solution of the first approximation [30] of the equation system (1), (2) taking into account (3), (4) (for FM AS relative variations of the amplitude $a(t, \tau)$ and absolute frequency variations $\omega(t, \tau))$ has the form:

$$
\begin{gathered}
a(t, \tau)=m_{\mathrm{AM}} f_{\mathrm{mod}}(t)+\Gamma(t, \tau) K_{\mathrm{a}} \cos [\delta(t, \tau)-\psi], \\
\omega(t, \tau)=\omega_{0}\left\{1+m_{\mathrm{FM}} f_{\mathrm{mod}}(t)-\Gamma(t, \tau) L_{\mathrm{a}} \sin [\delta(t, \tau)+\theta]\right\}
\end{gathered}
$$

where $K_{\mathrm{a}}, L_{\mathrm{a}}$ are autodyne amplification and frequency deviation coefficients, respectively; $\psi=\arctan (\rho), \quad \theta=$ $\arctan (\gamma)$ are phase displacement angles; $\rho=\varepsilon / Q_{\mathrm{L}}, \gamma=\beta / \alpha$ are non-isodromity and non-isochronity coefficients, respectively $[29,30]$. The latter terms in the right-hand side of (5) and (6) represent the formation of the autodyne response useful components caused by the reflected radiation impact.

To obtain analysis results of the autodyne response for the general case of arbitrary ratio of reflected radiation time delay $\tau$ and autodyne signal period $T_{\mathrm{a}}$, a well-known approach to the analysis of retarded systems is used. This approach is developed in [29] for the case of signal analysis of autodynes without modulation. It consists in expanding functions $A(t, \tau)$ and $\Psi(t, \tau)$ of the delayed impact into Tailor series with respect to a small parameter - ratio of $\tau$ to the current time $t$. Application of this approach assumes the absence of breaks of (5), (6) over all time interval of autodyne response formation. In addition, we exclude from consideration the transients in reverse zones of the modulation function, taking into account the strong inequality $\tau<<2 \pi / \Omega_{\text {mod. }}$. Taking into consideration all mentioned above, expressions for $\Gamma(t, \tau)$ and $\delta(t, \tau)$ in (5), (6) as functions of the normalized (dimensionless) delay time $\tau_{n}=\omega_{0} \tau / 2 \pi$ are as follows:

$$
\begin{gathered}
\Gamma\left(t_{n}, \tau_{n}\right)=\Gamma_{0}\left\{1+2 \pi r_{n} \Gamma_{0} K_{a} \sum_{m=0}^{M}(-1)^{m} \mathrm{X}_{m}\left(r_{n}\right) \times\right. \\
\left.\sin \left[\delta\left(t_{n}, \tau_{n}\right)-\psi+\Theta_{m}\left(r_{n}\right)\right]\right\}, \\
\delta\left(t_{n}, \tau_{n}\right)=2 \pi \tau_{n}+\pi B_{\mathrm{FM}} f_{\mathrm{mod}}\left(t_{n}\right)- \\
C_{F B} \sum_{m=0}^{M}(-1)^{m} \mathrm{X}_{m}\left(r_{n}\right) \sin \left[\delta\left(t_{n}, \tau_{n}\right)+\theta+\Theta_{m}\left(r_{n}\right)\right]
\end{gathered}
$$

where $r_{n}=l / \Lambda_{\mathrm{a}}=\tau / T_{\mathrm{a}}$ is the parameter of distance normalized to a wavelength of autodyne signal $\Lambda_{\mathrm{a}}=c T_{\mathrm{a}} / 2 ; B_{\mathrm{FM}}=$ $\Delta \omega_{\mathrm{FM}} \tau / 2 \pi$ is the parameter of 'FM base' determining the number of signal periods being stacked on the modulation function period in case of motionless reflecting object; $C_{\mathrm{FB}}=\Gamma_{0} L_{\mathrm{a}} \omega_{0} \tau$ is the feedback (FB) parameter of the autodyne system 'oscillator - reflecting object' [25]; $t_{n}=$ $\Omega_{\text {mod }} t / 2 \pi$ is the normalized time of the modulation function; $\mathrm{X}_{m}\left(r_{n}\right), \Theta_{m}\left(r_{n}\right)$ are module and phase of terms of series from (7) and (8):

$$
\begin{gathered}
\mathrm{X}_{m}\left(r_{n}\right)=\frac{\left(2 \pi r_{n}\right)^{2 m} \sqrt{4(m+1)^{2}+\left(2 \pi r_{n}\right)^{2}}}{2(m+1) \times(2 m+1) !}, \\
\Theta_{m}\left(r_{n}\right)=-\arctan \left[\pi r_{n} /(m+1)\right] .
\end{gathered}
$$

Autodyne variations of oscillation amplitude are usually small $\left(\Gamma_{0} K_{\mathrm{a}}<<1\right)$ and in (7) we can neglect the second term in large parenthesis assuming $\Gamma(t, \tau)=\Gamma_{0}$ in (5), (6). This approximation in the AS mathematical model assumes taking into account only the phase delay. The further analysis of AS response formation features will be performed on the basis of the expressions for autodyne amplitude characteristic (AAC) $a_{n}\left(t_{n}, \tau_{n}\right)$ and autodyne frequency characteristic (AFC) $\chi_{n}\left(t_{n}, \tau_{n}\right)$ extracted from (5) and (6):

$$
\begin{aligned}
& a_{n}\left(t_{n}, \tau_{n}\right)=a\left(t_{n}, \tau_{n}\right) / a_{m}=\cos \left[\delta\left(t_{n}, \tau_{n}\right)-\psi\right], \\
& \chi_{n}\left(t_{n}, \tau_{n}\right)=\chi\left(t_{n}, \tau_{n}\right) / \chi_{m}=-\sin \left[\delta\left(t_{n}, \tau_{n}\right)+\theta\right]
\end{aligned}
$$

where $a_{m}=\Gamma_{0} K_{\mathrm{a}}, \chi_{m}=\Gamma_{0} L_{\mathrm{a}}$ are maximal values of oscillator amplitude and frequency variations. These characteristics are determining for autodyne signal formation, therefore, they are called 'signals' in the theory of autodyne systems [29], [30].

The solution of the transcendent equation (8) on condition of its smoothness when $C_{\mathrm{FB}}<1$ can be obtained by the method of successive approximations. This solution in the form of the autodyne phase characteristic (APC) $\delta\left(t_{n}, \tau_{n}\right)$ has the form:

$$
\begin{aligned}
& \delta\left(t_{n}, \tau_{n}\right)=\delta\left(t_{n}, \tau_{n}\right)_{(0)}-C_{\mathrm{FB}} \sum_{m=0}^{M}(-1)^{m} \mathrm{X}_{m}\left(r_{n}\right) \times \\
& \sin \left[\delta\left(t_{n}, \tau_{n}\right)_{(1)}+\theta+\Theta_{m}\left(r_{n}\right)-\right. \\
& C_{\mathrm{FB}} \sum_{m=0}^{M}(-1)^{m} \mathrm{X}_{m}\left(r_{n}\right) \sin \left[\delta\left(t_{n}, \tau_{n}\right)_{(2)}+\theta+\Theta_{m}\left(r_{n}\right)-\ldots\right. \\
& \left.\left.-C_{F B} \sum_{m=0}^{M}(-1)^{m} \mathrm{X}_{m}\left(r_{n}\right) \sin \left[\delta\left(t_{n}, \tau_{n}\right)_{(k)}+\theta+\Theta_{m}\left(r_{n}\right)\right] \ldots\right]\right]
\end{aligned}
$$

where $\delta\left(t_{n}, \tau_{n}\right)_{(0,1, \ldots, k)}=2 \pi \tau_{n}+\pi B_{\mathrm{FM}} f_{\text {mod }}\left(t_{n}\right)$; the order of 
approximation is denoted by indices in parenthesis near terms $\delta\left(t_{n}, \tau_{n}\right)$.

The linear APC, which is typical for homodyne SRRs, corresponds to the zero approximation when only the first term in (13) $\delta\left(t_{n}, \tau_{n}\right)_{0}$ is taken into consideration. Further approximations add nonlinearity in this APC, which is an attribute of the autodyne SRRs. Therefore, in the current research the main attention will be focused on the revelation of AS signal features with the sinusoidal FM under conditions when the FB parameter $C_{\mathrm{FB}}$ is commensurable with unity. It usually takes place with the growth of values of reflected radiation level (the quantity $\Gamma_{0}$ ) and (or) the time delay $\tau$.

\section{Numerical Results}

FM AS signals are usually registered in the power source circuit (the auto-detection signal) by means of detecting its oscillation amplitude variations in the form of AAC $a_{n}\left(t_{n}, \tau_{n}\right)$ or output power variations [1, 12-14, 25]. The phase variations $\delta\left(t_{n}, \tau_{n}\right)$, which are caused by the modulation process and by the autodyne variations of frequency $\chi_{n}\left(t_{n}, \tau_{n}\right)$ as well as the reflecting object movement, contribute in the signal formation. Below we consider the case of fixed reflecting object assuming that $\tau_{n}=0$ and $\delta\left(t_{n}, \tau_{n}\right)=\delta\left(t_{n}\right), a_{n}\left(t_{n}, \tau_{n}\right)=a_{n}\left(t_{n}\right), \chi_{n}\left(t_{n}, \tau_{n}\right)=\chi_{n}\left(t_{n}\right)$. To reveal autodyne signal formation peculiarities the numerical method is used.

Figure 2 shows the APC timing diagrams $\delta\left(t_{n}\right)$, the instantaneous frequency difference $\operatorname{IFD}\left(t_{n}\right)=\mathrm{d} \delta\left(t_{n}\right) / \mathrm{d} t_{n}$, characterizing the variation speed of the phase incursion of the reflected radiation as well as AAC $a_{n}\left(t_{n}\right)$. Calculations of these characteristics were performed on the basis of (11), (13) for the modulation function $f_{\bmod }\left(t_{n}\right)=\sin \left(2 \pi t_{n}\right)$ for different $r_{n}$. Spectra of signal characteristics AAC $a_{n}\left(F_{n}\right)$ for the cases shown in Fig. 2 are presented in Fig. 3., where $F_{n}$ is a frequency of autodyne signal normalized to $\Omega_{\text {mod. }}$.

Here and further, calculations were performed at $\theta=1, \quad \psi=0.2, \quad B_{\mathrm{FM}}=5.3, \quad C_{\mathrm{FB}}=0.8, \quad \Omega_{\mathrm{mod}}=2 \pi ;$ $k=M=50$. Below we shall define 'the operation zone' as the segment of the distance equal to a unit of the dimensionless normalized distance $r_{n}$ beginning with the first operation zone where $0 \leq r_{n} \leq 1$. It is necessary to note that numbers $M$ and $k$ in (8), (13), which were taken for calculations, ensure the convergence of calculation results in the ranges $r_{n} \leq 5$ and $C_{\mathrm{FB}} \leq 0.98$.

Diagrams in Fig. 2 show that in this case there are no signal phase jumps by contrast with the case of applying the linear modulation functions in AS [24], [31], [32]. However, while using the sinusoidal modulation function, the phase variations of the autodyne response with the frequency $\Omega_{\text {mod }}$ and the modulation index $\pi B_{\mathrm{FM}}$ are observed. This modulation causes appearance of additional components in the signal spectrum (see Fig. 3(c)). These signal peculiarities are in agreement with the theory of ope-

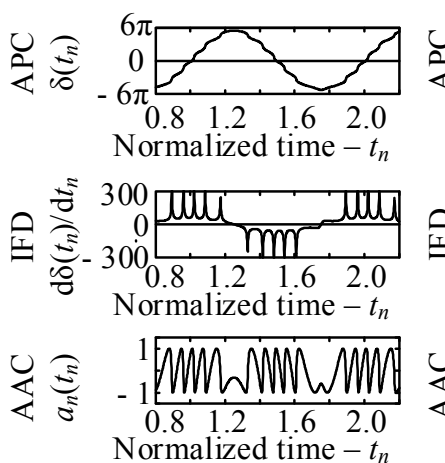

(a)

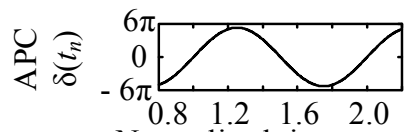

Normalized time $-t_{n}$

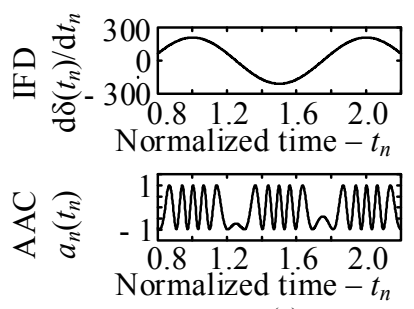

(c)
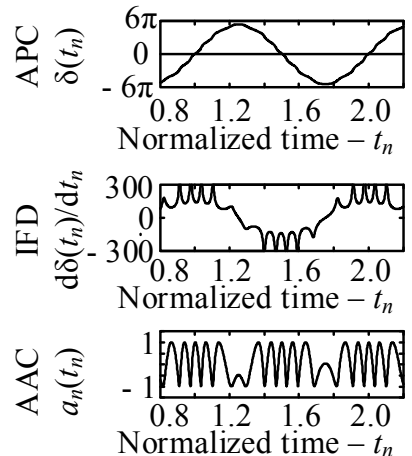

(b)

Normalized time $-t_{n}$

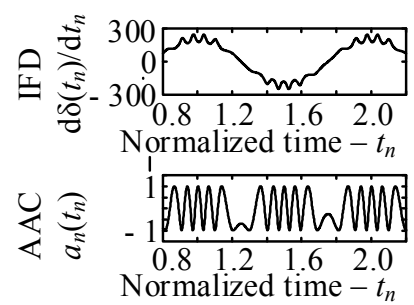

(d)

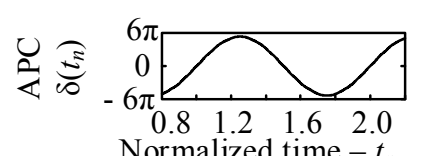

Fig. 2. Diagrams of APC $\delta\left(t_{n}\right), \operatorname{IFD}\left(t_{n}\right)$ and $\mathrm{AAC} a_{n}\left(t_{n}\right)$, calculated for different $r_{n}$ : (a) $r_{n}=0$, (b) $r_{n}=0.5$, (c) $r_{n}=1$, (d) $r_{n}=1.5$.

ration of homodyne systems, but the presence of signal anharmonic distortions substantially distinguishes autodyne systems from homodyne ones. As diagrams in Fig. 2(a) show, these distortions look like variations of 'wave slope' of signal characteristics AAC $a_{n}\left(t_{n}\right)$ when the sign of the derivative of the FM modulation function changes.

The analysis of timing and spectral diagrams in Figs 2(a) and 3(a) shows that results presented in the current paper for the case of $r_{n}=0$ completely correspond to the results of earlier FM AS signal research described in [24-27]. In these publications, the signal analysis was performed only for the first approximation of retarded impact functions when $\tau<<T_{\mathrm{a}}$. It is shown that phase shift irregularity $\delta\left(t_{n}\right)$ (at $C_{\mathrm{FB}}$ commensurable with unity) causes anharmonic distortions of signal characteristics and appearance of higher harmonic components.

Diagrams in Fig. 2(b)-(d) show that as $r_{n}$ grows the derivative $\operatorname{IFD}\left(t_{n}\right)$ peak height and the degree of anharmonic distortions of AAC essentially decrease. Such a tendency is especially noticeable in the first operation zone where $0 \leq r_{n} \leq 1$. Calculations of characteristics for other $r_{n}$ values show that in the case when $r_{n}$ is divisible by the integer number (i.e. $r_{n}=1,2, \ldots$ ) AAC have almost sinusoidal form without 'wave slopes' and higher harmonics are absent in their spectra (see Fig. 3(c)). When $C_{\mathrm{FB}}<<1$ signal characteristics $a_{n}\left(t_{n}\right)$ also have no peculiarities since autodyne signals being formed completely correspond to signals of homodyne FM systems, as we already mentioned. 


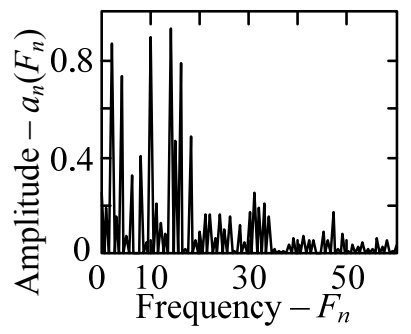

(a)

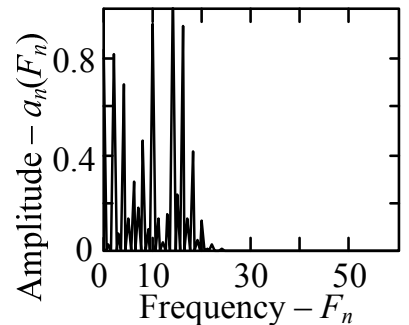

(c)

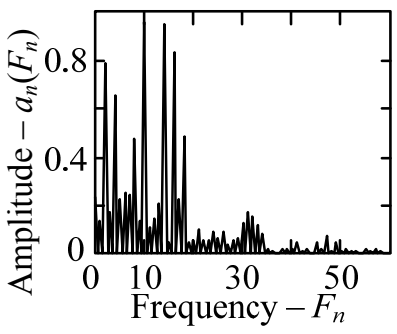

(b)

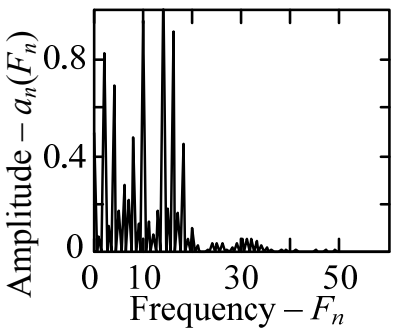

(d)
Fig. 3. Spectra $a_{n}\left(F_{n}\right)$ calculated for different $r_{n}$ : (a) $r_{n}=0$, (b) $r_{n}=0.5$, (c) $r_{n}=1$, (d) $r_{n}=1.5$.

It is necessary to mention that the character of the 'continuous' AS signal spectrum for the sinusoidal FM (see Fig. 3) substantially differs from the discrete spectrum obtained at linear FM types [24], [32]. For sinusoidal FM the effective spectrum width is $\pi$-times more than its value in comparison with the case of the non-symmetric sawtooth FM law and is $\pi / 2$-times more in comparison with the case of the symmetric saw-tooth law [24]. These differences are caused by nonlinearity of signal phase modulation and hence larger value of modulation curve slope at sinusoidal modulation function than for the case of linear FM laws.

The most important point which follows from the analysis of spectra presented in Fig. 3(b)-(d) consists in the fact that as $r_{n}$ grows the level of higher harmonic components substantially decreases. For better representation of this dependence we use a generalized parameter characterizing the distortion degree of quasi-periodic oscillations called the total harmonic distortion (THD) coefficient. Calculation results of the THD using the first 10 terms of the Fourier series (as functions of $r_{n}$ ) are presented in Fig. 4(a) for different values of $C_{\mathrm{FB}}$.

Plots in Fig. 4(a) show that the largest signal distortions are observed in the middle of the first operation zone. If $C_{\mathrm{FB}}=0.8$, the THD achieves $60 \%$. With further transition into operation zones of higher order, signal distortions substantially decrease achieving minimal values in the case when the $r_{n}$ value is equal to an integer number (i.e. $r_{n}=$ $1,2, \ldots)$. It is necessary to note that the THD in case of the sinusoidal modulation is much higher than in cases of linear FM laws [32].

Returning to the analysis of calculation results presented in Fig. 3, it is necessary to mention the presence of DC components $a_{0}\left(F_{n}\right)$ in spectra. Taking this phenomenon into account is important for signal processing and noiseimmunity analysis of systems.

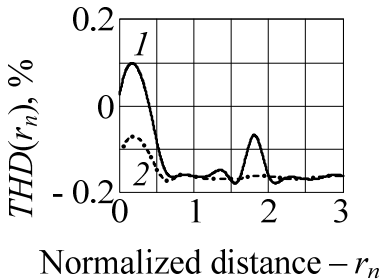

(a)

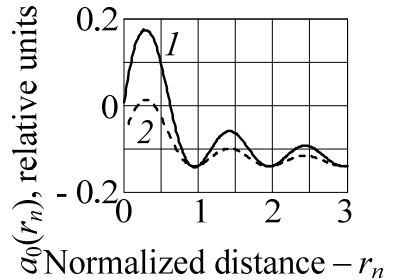

(b)
Fig. 4. Plots $T H D\left(r_{n}\right)(\mathrm{a})$ and $\mathrm{DC}$ components $a_{0}\left(r_{n}\right)(b)$, calculated for $C_{\mathrm{FB}}=0.8$ (curve 1$)$ and $C_{\mathrm{FB}}=0.4$ (curve 2).

Calculation results in the form of $a_{0}\left(r_{n}\right)$ are presented in Fig. 4(b). These curves show that at rather large values of $C_{\mathrm{FB}}$ the level of DC component may achieve $10 \ldots 15 \%$ from autodyne variation amplitudes $a_{m}$. If $C_{\mathrm{FB}}<<1$ these components in the FM AS output signals are almost absent.

To explain the character of the functions obtained above, it is necessary to introduce the notions of the equivalent FB parameter $C_{\text {eq }}$ and the angle of dynamic displacement $\Delta \delta_{\mathrm{DD}}$ of autodyne response phase: $C_{\mathrm{eq}}=C_{\mathrm{FB}} K_{\mathrm{DF}}$ and $\Delta \delta_{\mathrm{DD}}=\delta\left(r_{n}\right)-\delta\left(r_{n}=0\right)$. Here $K_{D F}=\sum_{m=0}^{M}(-1)^{m} \mathrm{X}_{m}\left(r_{n}\right)$ is the 'dynamic factor' of FB included in (8) and (12); $\delta\left(r_{n}\right)$, $\delta\left(r_{n}=0\right)$ are phases of the instantaneous reflection factor obtained at the current value of $r_{n}$ and at its zero value, respectively. Calculation results for $K_{\mathrm{DF}}\left(r_{n}\right)$ and $\Delta \delta_{\mathrm{DD}}\left(r_{n}\right)$ are presented in Fig. 5; for the function $\Delta \delta_{\mathrm{DD}}\left(r_{n}\right)$ two curves are obtained: curve 1 is for the non-isochronic oscillator, and curve 2 is for the isochronic one.

The curve $K_{\mathrm{DF}}\left(r_{n}\right)$ (Fig. 5(a)) shows that as $r_{n}$ grows in the first operation zone (where $0<r_{n}<1$ ) $K_{\mathrm{DF}}$ and, accordingly, $C_{\mathrm{eq}}$ decrease almost by the order. Then, with $r_{n}$ growth, $C_{\text {eq }}$ asymptotically damps with slight increases in the middle part of operation zones of higher order where $r_{n}>1$.

With $r_{n}$ growth in the first operation zone, dynamic phase variations $\Delta \delta_{\mathrm{DD}}$ of signal characteristics are also the largest (see Fig. 2(a) and (b)). At $r_{n}=1$ there is the first minimum of this function which is determined by the value of $\theta$ characterizing the oscillator non-isochronity (see Fig. 5(b)). With further $r_{n}$ growth, the asymptotical damping is also observed for 'oscillations' of dynamic phase variations $\Delta \delta_{\mathrm{DD}}$.

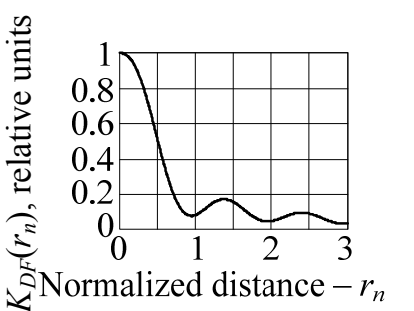

(a)

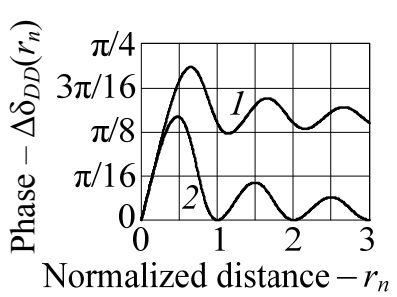

(b)
Fig. 5. Plots of the dynamic FB factor $K_{\mathrm{DF}}\left(r_{n}\right)$ (a) and the phase displacement $\Delta \delta_{\mathrm{DD}}\left(r_{n}\right)$ (b) of signals: $\theta=1$ (curve 1 ), $\theta=0$ (curve 2). 
To understand the physical sense of the revealed dependence of FM AS signal formation, let us consider the simplified model of the interaction process of the autodyne oscillator with the proper reflected radiation using the stepping method on the example of radio-pulse autodyne (see [33]). This model research results show that with shortening of the relative radio-pulse duration (which is equivalent to the normalized distance $r_{n}$ growth), the number of partial reflections decreases during radio-pulse impact. This causes the reduction of the FB parameter $C_{\mathrm{eq}}$ of the autodyne system and, accordingly, the signal distortion level. Achieving the value of normalized distance $r_{n}=1$ (when the reflected radiation impact becomes single-partial) provides forming almost sinusoidal autodyne variations of oscillation amplitude and frequency.

\section{Experimental Results}

Oscillation module based on the 8-mm wavelength range Gunn diode was used to perform experimental research of FM AS signal peculiarities. The module is integrated in the AS, which was developed for checking the occupation of point-works on the hump-yards [6], [23]. The general view of AS and its main components are presented in Fig. 6. An antenna with the millimeter-wave-

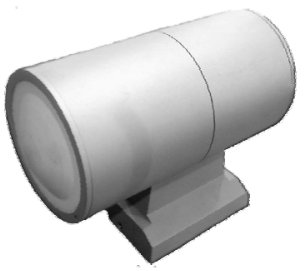

(a)

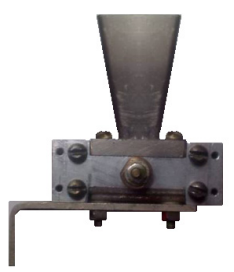

(b)

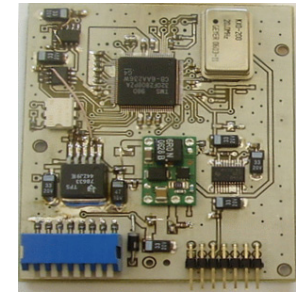

(c)
Fig. 6. General view of AS (a), of autodyne module with the horn antenna (b) and the printed board (c) of the control and signal processing unit.

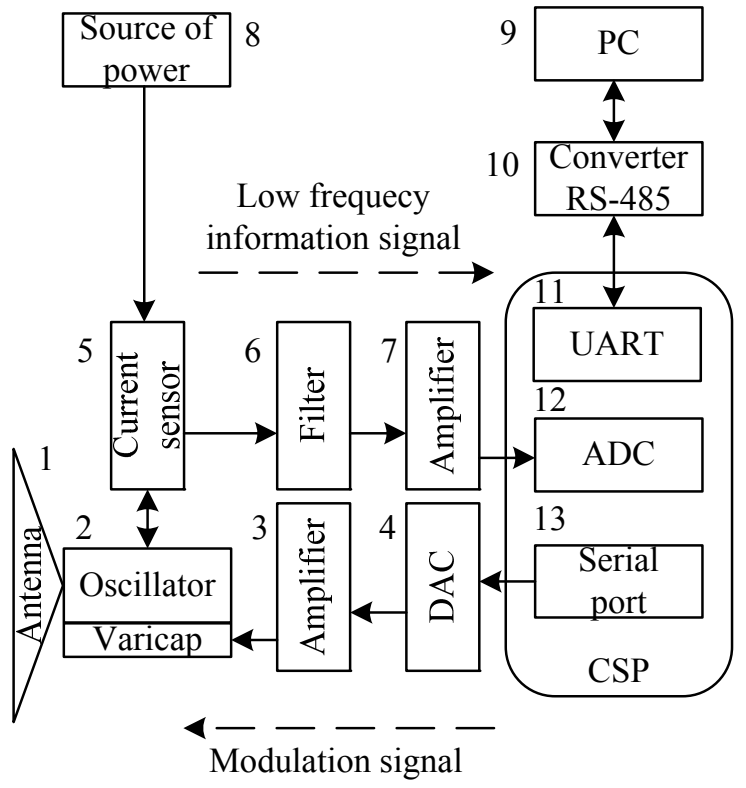

Fig. 7. Structural diagram of AS. length module and the printed circuit board with modules of probing signal generation, digital signal processing and communication RS-485 interface are encapsulated into the cylindrical hermetic case made of the duralumin with a diameter of $92 \mathrm{~mm}$ and a length of $170 \mathrm{~mm}$. The dielectric lens of antenna is simultaneously the front wall of the case.

The autodyne oscillator of AS is made on the basis of discrete components: the Gunn diode AA727A and the varicap 3A637A-6. The effective radiated power is $25 \mathrm{~mW}$, the central frequency is $36.5 \mathrm{GHz}$. The oscillator provides the $500 \mathrm{MHz}$ frequency deviation, the modulation frequency is $10 \mathrm{kHz}$. The antenna type is the horn-lens with the beamwidth $6 \times 6$ degrees.

The structural diagram of AS is presented in Fig. 7. The central signal processor CSP which simultaneously performs functions of FM law generation and the primary processing of signals converted by the autodyne is based on the digital signal processor TMS320F2808. The following hard-ware function blocks of DSP are used: the analog-to-digital converter ADC (12 in Fig. 7); the transceiver of SPI-bus (13) which controls digital-to-analog converter DAC (4); the transceiver of SCI-bus (11) which performs the data exchange with the indicator and control units; the high-speed computing kernel performing all the functions of digital signal processing (the spectral analysis, digital signal filtering, the signal analysis and data formation for indication). The DAC unit (4) is intended to form modulation signal which is applied through the amplifier (7) to the varicap (included in the UHF oscillator (2)) performing FM.

UHF FM signal is emitted by the antenna system (1) of the transceiver module (2). Electromagnetic radiation reflected from the target passes back into the UHF oscillator (2) and causes the variations of oscillation parameters. The autodyne signal is registered by the current sensor (5) which converts the current variations in the power supply circuit of the Gunn oscillator into the output voltage. After passing through the filter (6) and the amplifier (7), this signal is applied to ADC (12) of CSP.

To exclude received signal components providing no useful information (caused by PAM and reflection from near objects) preliminary signal filtering is used. For this purpose the algorithm of the 'moving average' is applied to input signal; the algorithm acts as a low-pass filter [34], [35]. After low-frequency components extraction they are subtracted from the initial signal. It results in the formation of the 'dead zone' near the AS.

If there is a reflecting object in the AS operation zone, there is a signal at the output of the preliminary filter. Further, the main frequency selection algorithm is applied to this signal in the filter-spectrum analyzer which is performed on the basis of the FFT function.

The further digital processing of the signal components remained at the main filter output consists in determination of the mean number of signal periods over one 


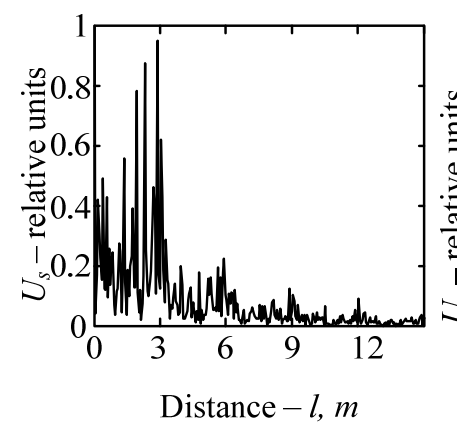

(a)

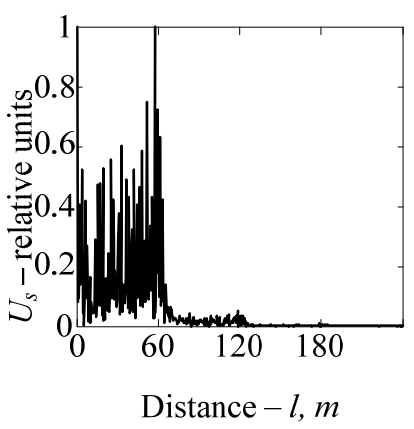

(b)
Fig. 8. Spectra of signal converted by the autodyne obtained from the corner reflector $(a)$ and from the wall of fivefloor building $(b)$.

period of modulation function. After multiplication by the calibration factor, the result is the distance to the object. A half of the difference of information signal period number on the ascending and descending parts of the modulation function, taking into account the calibration factor, provides determination of the relative velocity and the target direction. The latter is obtained on the basis of the difference result sign.

The analysis results are transferred through the universal asynchronous receiver-transmitter UART (11) and the converter RS-485 (10) to the PC (9) which is used as the imaging system for radar information.

Figure 8 shows the signal spectra obtained at the output of the SRR processing unit from the corner reflector (a) and from the wall of the five-floor building (b). The corner reflector with scattering cross-section about $10 \mathrm{~m}^{2}$ was mounted at the distance $l=3 \mathrm{~m}$ (delay time $\tau=2 \times 10^{-8} \mathrm{~s}$ ) from the antenna aperture. The building wall was situated at the distance $l=60 \mathrm{~m}\left(\tau=4 \times 10^{-7} \mathrm{~s}\right)$. The largest frequency of the converted signal from the corner reflector was about $314 \mathrm{kHz}\left(T_{\mathrm{a}}=3.2 \times 10^{-6} \mathrm{~s}\right)$, while from the building wall $-6.3 \mathrm{MHz}\left(T_{\mathrm{a}}=1.6 \times 10^{-7} \mathrm{~s}\right)$. Signal amplitudes $\left(U_{s}\right)$ in both cases were practically the same and FB parameter $C_{\mathrm{FB}} \approx 0.8$.

In the first case (Fig. 8(a)), there are noticeable levels of the second and third harmonics in the spectrum, which is typical for anharmonic distortions of autodyne signals. In the second case, higher harmonic components have substantially lower levels than in the first case. Comparison of spectra in Fig. 8(a), (b) and spectra presented in Fig. 3(a), (d) shows their qualitative agreement. It let us conclude that obtained experimental data confirm the adequacy of the above-developed mathematical model for the analysis and calculation of signal and spectral characteristics of FM AS.

\section{Conclusions}

Features of FM AS signal formation are considered for sinusoidal FM law in cases of various ratio of the delay time of the reflected signal $\tau$ and the autodyne signal period
$T_{\mathrm{a}}$. Result analysis of the performed research shows that the frequency of the signal converted by the autodyne in the whole range of distances to the target object exactly corresponds to the frequency of signal obtained in the case of the homodyne system [1-4], [9-11]. It is proved that at small distances to the reflected object when $\tau<<T_{\mathrm{a}}$ the obtained analysis results conform to results of previous investigations [24-27]. When $C_{\mathrm{FB}}$ is commensurable with unity, substantial distortions of autodyne signals and appearance of higher harmonic components in the signal spectrum are observed. It requires additional consideration in signal processing devices.

When the distance to the reflecting object grows, delay time of the reflected radiation $\tau$ becomes equal or more than the autodyne signal period $T_{\mathrm{a}}$. In this case, the anharmonic distortion degree of AAC $a_{n}\left(t_{n}\right)$ and AFC $\chi_{n}\left(t_{n}\right)$ substantially decreases. The distortion reduction is especially noticeable at distances divisible by the integer number of $\tau / T_{\mathrm{a}}=r_{n}$ (i.e. $\left.r_{n}=1,2, \ldots\right)$.

The analysis results obtained in this paper develop and expand the results of the known investigations published in [29] when dealing with FM in oscillators as well as the results of investigations published in [24], [31] when dealing with consideration (in the autodyne model) of phase variation dynamics of reflected radiation in the process of its propagation to the reflecting object and back. The dependence of FM AS signal formation is revealed on the example of UHF oscillator application. They have general character and physical interpretation on the basis of the stepping method known from the theory of retarded systems. Thus, the obtained results, in our opinion, can be also used for signal calculation of AS made on the basis of semiconductor laser modules [25-27].

\section{Acknowledgments}

The present paper is prepared in accordance with the Agreement on scientific-technological cooperation between UrFU, IRE NASU and NRU 'MPEI' as well as at financial support of Russian Federation Government, order No. 211, contract No. 02.A03.21.0006.

\section{References}

[1] KOMAROV, I. V., SMOLSKIY, S. M. Fundamentals of ShortRange FM Radar. Norwood: Artech House, 2003. ISBN 1-58053110-5. DOI: 10.1109/MAES.2004.1346903

[2] YANOVSKY, F. J. Millimeter-Wave Radar: Principles and Applications. Chapter 10 from book: XIAO, S.-Q., ZHOU, M.-T., ZHANG, Y. (eds.) Millimeter Wave Technology in Wireless PAN, $L A N$, and MAN. New York: CRC, 2008, 448 p (p. 305-375). ISBN-13: 978-0-8493-8227-7

[3] CHARVAT, G. L. Small and Short-Range Radar Systems. CRC Press, 2014. 430 p. ISBN-10: 143986599X 
[4] SAPOnARA, S., GRECO, M., RAGONESE, E., et al. Highly Integrated Low-power Radars. Boston, London: Artech House Publishers, 2014, 215 p. ISBN: 978-1-60807-665-9

[5] ARMStrong, B. M., BROWN, R., RIX, F., et al. Use of microstrip impedance-measurement technique in the design of a BARITT diplex Doppler sensor. IEEE Transactions on Microwave Theory and Techniques, 1980, vol. MTT-28, no. 12, p. 1437-1442. DOI: $10.1109 /$ TMTT.1980.1130263

[6] ERMAK, G. P., POPOV, I. V., VASILEV, A. S., et al. Radar sensors for hump yard and rail crossing applications. Telecommunication and Radio Engineering, 2012, vol. 71, no. 6, p. 567-580. DOI: 10.1615/TelecomRadEng.v71.i6.80

[7] BORIC-LUBECKE, O., DROITCOUR, A. D., LUBECKE, V. M., PARK, B.-K., SINGH, A. (eds.) Doppler Radar Physiological Sensing. New York: John Wiley \& Sons, 2016. 304 p.

[8] AYHAN, S., THOMAS, S., KONG, N., et al. Millimeter-wave radar distance measurements in micro machining. In IEEE Topical Conference on Wireless Sensors and Sensor Networks (WiSNet). San Diego (CA, USA), 2015, p. 65-68. DOI: 10.1109/WISNET.2015.7127413

[9] ANGHEL, A., VASILE, G., CACOVEANU, R., et al. Short-range wideband FMCW radar for millimetric displacement measurements. IEEE Transactions on Geoscience and Remote Sensing, 2014, vol. 52, no. 9, p. 5633-5642. DOI: 10.1109/TGRS.2013.2291573

[10] STURDIVANT, R., HARRIS, M. Transmit Receive Modules for Radar and Communication Systems. Artech House, 2015, 282 p. ISBN-10: 1608079791

[11] PIPER, S. O. Homodyne FMCW radar range resolution effects with sinusoidal nonlinearities in the frequency sweep. In Proceedings International Radar Conference. Alexandria (VA, USA), 1995, p. 563-567. DOI: 10.1109/RADAR.1995.522609

[12] ALIDOOST, S. A., SADEGHZADE, R., FATEMI, R. Autodyne system with a single antenna. In Proceedings of the 11-th International Radar Symposium (IRS 2010). Vilnius (Lithuania), 2010, p. 406-409.

[13] KIM, S., KIM, B.-H., YOOK, J.-G., YUN, G.-H. Proximity vital sign sensor using self-oscillating mixer. In URSI Asia-Pacific Radio Science Conference (URSI AP-RASC). Seoul (South Korea), 2016, p. 1446-1448. DOI: 10.1109/URSIAP-RASC.2016.7601402

[14] USANOV, D. A., POSTELGA, A. E. Reconstruction of complicated movement of part of the human body using radio wave autodyne signal. Biomedical Engineering, 2011, vol. 45, no. 1, p. 6-8. DOI: 10.1007/s10527-011-9198-9

[15] NAGASAKU, T., KONDOH, H., SHUNODA, H. Radar Sensor. Patent US 6717544 B2. Pub. Date: April, 6, 2004. Filed: Aug. 26 , 2002.

[16] VOTOROPIN, S. D. Autodyne sensors of the EHF range on Gunn diodes. In 38th European Microwave Conference. Amsterdam (The Netherlands), 2008, p. 1330-1333. DOI: 10.1109/EUMC.2008.4751709

[17] UTAGAWA, H., MATSUI, T. Microwave/Millimeter Wave Sensor. Patent Appl. Publ. US 2010/0117891 A1. Pub. Date: May 13, 2010. Filed: Mar. 31, 2008.

[18] EFANOV, A. A., DiskUS, C. G., STElzER, A., et al. Development of a low-cost $35 \mathrm{GHz}$ radar sensor. Annals of Telecommunications, 1997 , vol. 52, no. 3, p. 219-223. DOI: 10.1007/BF02996047

[19] GIULIANI, G., NORGIA, M., DONATI, S., BOSCH, T. Laser diode self-mixing technique for sensing applications (Review article). Journal of Optics A: Pure and Applied Optics, 2002, vol. 4, no. 6, p. 283-294. DOI: 10.1088/1464-4258/4/6/371
[20] USANOV, D. A., SKRIPAL, AL. V., SKRIPAL, AN. V., et al. A microwave autodyne meter of vibration parameters. Instruments and Experimental Techniques, 2004, vol. 47, no. 5, p. 689-693. DOI: 10.1023/B:INET.0000043882.16801.3a

[21] JEFFORD, P. A., HOWES, M. S. Modulation schemes in low-cost microwave field sensor. IEEE Transactions of Microwave Theory and Technique, 1985, vol. MTT-31, no. 8, p. 613-624. DOI: 10.1109/TMTT.1983.1131559

[22] VOTOROPIN, S. D., NOSKOV, V. YA. Analysis of operating regimes of EHF hybrid-integrated autodynes based on the Gunn micro power mesa planar diodes. Russian Physics Journal, 2002, vol. 45, no. 2, p. 195-206. DOI : 10.1023/A:1019664300993

[23] VARAVIN, A. V., VASILIEV, A. S., ERMAK, G. P., POPOV, I. V. Autodyne Gunn-diode transceiver with internal signal detection for short-range linear FM radar sensor. Telecommunication and Radio Engineering, 2010, vol. 69, no. 5, p. 451-458. DOI: 10.1615/TelecomRadEng.v69.i5.80

[24] VOTOROPIN, S. D., NOSKOV, V. YA., SMOLSKIY, S. M. An analysis of the autodyne effect of oscillators with linear frequency modulation. Russian Physics Journal, 2008, vol. 51, no. 6, p. 610-618. DOI: $10.1007 / \mathrm{s} 11182-008-9083-5$

[25] GIULIANI, G., NORGIA, M., DONATI, S., BOSCH, T. Laser diode self-mixing technique for sensing applications (Review article). Journal of Optics A: Pure and Applied Optics, 2002, vol. 4, no. 6, p. 283-294.

[26] USANOV, D. A., SKRIPAL, A. V., ASTAKHOV, E. I., DOBDIN S. YU. Autodyne interferometry for range-finding under laser radiation wavelength modulation. Technical Physics Letters, 2016, vol. 42, no. 9, p. 919-922. DOI: 10.1134/S1063785016090121

[27] SOBOLEV, V. S., KASHCHEEVA, G. A. Self-mixing frequencymodulated laser interferometry. Optoelectronics, Instrumentation and Data Processing, 2008, vol. 44, no. 6, p. 519-529. DOI: $10.3103 / \mathrm{S} 8756699008060058$

[28] KUROKAWA, K. Injection locking of microwave solid-state oscillators. Proceedings of the IEEE, 1973, vol. 61, no. 10, p. 1386-1410. DOI: 10.1109/PROC.1973.9293

[29] NOSKOV, V. YA., IGNATKOV, K. A. Autodyne signals in case of random delay time of the reflected radiation. Telecommunication and Radio Engineering, 2013, vol. 72, no. 16, p. 1521-1536. DOI: 10.1615/TelecomRadEng.v72.i16.70

[30] NOSKOV, V. YA., IGNATKOV, K. A. About applicability of quasi-static method of autodyne systems analysis. Radioelectronics and Communications Systems, 2014, vol. 57, no. 3, p. 139-148. DOI: $10.3103 / \mathrm{S} 0735272714030054$

[31] IGNATKOV, K. A., VASILIEV, A. V. Signals of the autodyne FM radar for mm-wavelength range. In Proceedings of the 26-th International Crimean Conference "Microwave \& Telecommunication Technology" (CriMiCo'2016). Sevastopol, 2016, vol. 10, p. 2139-2145

[32] NOSKOV, V. YA., IGNATKOV, K. A., CHUPAHIN, A. P., et al. Peculiarities of signal formation of the autodyne short-range radar with linear frequency modulation. Visnik NTUU KPI, Seriia Radiotehnika. Radioaparatobuduvannija, 2016, no. 67, p. 50-57.

[33] NOSKOV, V. YA., IGNATKOV, K. A. Dynamics of autodyne response formation in microwave generators. Radioelectronics and Communications Systems, 2013, vol. 56, no. 5, p. 227-242. DOI: $10.3103 / \mathrm{S} 0735272713050026$

[34] HUSSAIN, Z. M., SADIK, A. Z., O'SHEA, P. Digital Signal Processing: An Introduction with MATLAB and Applications. Springer, 2011, 350 p. ISBN: 3642155901

[35] DANIELS, D. J. EM Detection of Concealed Targets. Wiley-IEEE Press, 2010, 284 p. ISBN 978-0-470-12169-6 


\section{About the Authors ...}

Vladislav Ya. NOSKOV was born in 1948. Graduated from the Radio Engineering Faculty of Tomsk Institute of Automated Control Systems and Radio Electronics in 1973. He received the Ph.D. degree in 1985, and the Dr.Sci. degree in 1995. At present, he is a professor of Technologies and Communication Means Dept. of Ural Federal University. The author of more than 300 scientific publications and 15 patents.

Kirill A. IGNATKOV was born in 1988. Graduated from the Institute of Radioelectronics and Information Technologies of Ural Federal University in 2010. Ph.D. thesis was defended in 2014. He works as the leading engineer of Technologies and Communication Means Dept. of Ural Federal University. The author of more than 100 scientific publications and 4 patents.

Andrey P. CHUPAHIN was born in 1992. Graduated from the Institute of Radioelectronics and Information Technologies of Ural Federal University in 2014 and currently is the $2^{\text {nd }}$-year $\mathrm{PhD}$-student at Technologies and Communication Means Dept. of Ural Federal University. The author of 16 scientific publications.

Aleksandr S. VASILYEV was born in 1979. Graduated from the Faculty of Radio-Physics of Kharkiv National University in 2001. Works as a junior scientific researcher in the Institute of Radio-Physics and Electronics of National Academy of Sciences of Ukraine. Developer of millimeter-wave surveillance radars and autodyne radar sensors for railway applications with digital signal processing. The author of more than 40 scientific publications.

Gennadiy P. ERMAK was born in 1946. Graduated from the Faculty of Radio-Physics of Kharkiv National University in 1968. He received the Ph.D. degree in 1984. Works as a senior scientific researcher and the Head of the Lab in the Institute of Radio-Physics and Electronics of National Academy of Sciences of Ukraine. Developer of radars and high resolution measurement systems based on millimeter wave autodyne and synchronized sources with digital signal processing. The author of more than 150 scientific publications and 3 patents.

Sergey M. SMOLSKIY was born in 1946. Graduated from the Radio Engineering Faculty of National Research University 'Moscow Power Engineering Institute' in 1970. He received the degree of Ph.D. in 1974 and the degree of Dr.Sci. in 1993. At present, he is a professor of Radio Signals Formation and Processing of National Research University 'Moscow Power Engineering Institute', Deputy Director of Institute of Radio Engineering and Electronics of MPEI. The author of more than 300 scientific publications, 15 scientific books and 8 patents. 